\title{
Simultaneous Subtotal Petrosectomy and Cochlear Implantation in Patients with a Cochlear Fistula
}

\author{
Jung Woo Lee, $\mathrm{MD}^{1}$, Ilyoung Cho, $\mathrm{MD}^{1}$, Soo-Keun Kong, $\mathrm{MD}, \mathrm{PhD}^{1,2}$, and Se-Joon $\mathrm{Oh}, \mathrm{MD}, \mathrm{PhD}^{1,2}$ \\ ${ }^{1}$ Department of Otorhinolaryngology and Biomedical Research Institute, Pusan National University Hospital; and \\ ${ }^{2}$ Department of Otorhinolaryngology, College of Medicine, Pusan National University, Busan, Korea
}

\begin{abstract}
- ABSTRACT -
Although chronic otitis -media (COM) with cochlear fistula is uncommon situation in cochlear implantation (CI), there are still debate on the choice between sequential and simultaneous COM management and CI. To decide one stage or staged operation could be one of an important consideration for operation preparation. We report two deaf patients with a cochlear fistula who simultaneously underwent subtotal petrosectomy, fistula repair, and CI. (J Clinical Otolaryngol 2020;31:196-199)
\end{abstract}

KEY WORDS: Cochlear implants $\cdot$ Chronic otitis media $\cdot$ Cochlear fistula.

\section{Introduction}

Chronic otitis media (COM) can cause sensorineural hearing loss and chronic otorrhea, and thus, may be the cause of deafness, requiring a cochlear implant (CI). ${ }^{1)}$ Although CI for COM is still debated on the choice between sequential and simultaneous COM management and CI, subtotal petrosectomy with $\mathrm{CI}$ has been commonly performed for inactive COM. ${ }^{2,3)}$ The presence of a cochlear fistula is rare in COM, with only a few patients reported to date. ${ }^{4)}$ Moreover, only one paper of CI has been reported in two patients with a cochlear fistula. ${ }^{5)}$ There have been no reports on simultaneous subtotal petrosectomy, fistula repair, and CI for COM with a cochlear fistula. Herein, we describe two deaf patients with a cochlear fistula who simultaneously underwent subtotal petrosectomy, fistula repair, and CI. This study was approved by the Institutional Review Board of Pusan National University Hospital (H-1909-025-083).

\section{Case Report}

\section{Case 1}

A 75-year-old woman presented with bilateral hearing disturbance. Hearing loss was congenital in the right ear and had developed a year before in the left ear. She was totally deaf in both ear at the time of presentation. On endoscopy, both the tympanic membranes were completely retracted. Computed tomography (CT) showed bilateral COM with left cochlear fistula around the promontory (Fig 1A). Magnetic resonance imaging (MRI) showed no abnormalities in the inner ear or cochlear nerve (Fig 1B). We decided to perform CI on the left side owing to the short duration of deafness. Electrode (FLEX ${ }^{28}$, MED-EL, Austria) was selected as straight type. Since there had been no otorrhea in the past year, subtotal petrosectomy with CI of the left ear and repair of the cochlear fistula with cartilage were conducted simultaneously. Electrodes were fully in- 
serted via the round window (Fig. 1C, and D). Twelve months after the surgery, the patient showed a category of performance (CAP) score of 6 and 76\% discrimination in the open-set sentence-recognition test. No signs of meningitis or recurrent COM was observed at postoperative 12 months.

\section{Case 2}

A 76-year-old woman presented with a 20 -year his- tory of bilateral hearing disturbance and intermittent otorrhea. Hearing loss had developed 20 years before in the right ear, and the patient had been using a hearing aid in the left ear. For the past 6 months, she complained about her worsened hearing ability of left ear, despite the use of hearing aids. On endoscopy, the right tympanic membrane showed a large perforation, the left tympanic membrane a small perforation, with no otorrhea in either ear. Pure tone audiometry showed bilater-
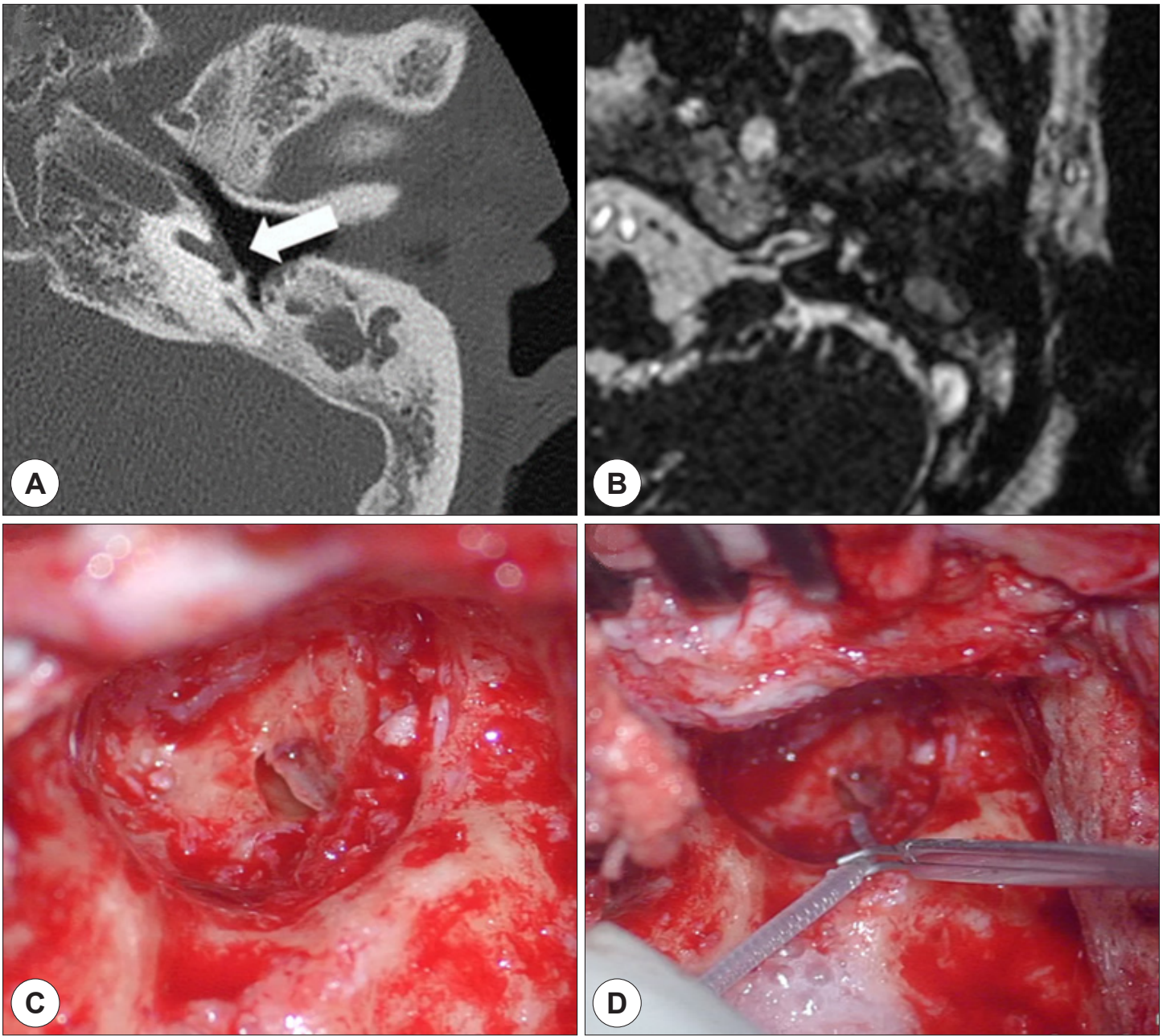

Fig. 1. Preoperative imaging and Intraoperative finding in case 1. A : Axial CT scan showing cochlear fistula. White arrow indicates the cochlear fistula. B : Magnetic resonance imaging (MRI) showed no abnormalities in the left inner ear or cochlear nerve. C : Cochlear fistula after removal of mucosa of middle ear. D : Electrode insertion via round window. 
al deaf status, a CAP score of 0 , and $0 \%$ discrimination in the open-set sentence-recognition test. Although MRI showed no abnormalities in the inner ear or cochlear nerve, temporal CT showed a cochlear fistula (Fig. 2A). Magnetic resonance imaging (MRI) showed no abnormalities in the inner ear or cochlear nerve (Fig 2B). Due to the difference in the duration of deafness between the ears, we decided to perform CI in the left ear. Straight electrode (Nucleus 522 Slim Straight, Nucleus, Austra- lia) was used for electrode full insertion because there was no residual hearing. The surgery was planned and performed similarly as case 1 , but the cochlear fistula was as small in size as the diameter of the electrode and located close to the round window. Therefore, the electrode was fully inserted via the cochlear fistula, and subsequently, closed with muscle tissues and glue (Fig. 2C, D). Twelve months after the surgery, the CAP score was 7 , and the open-set sentence-recognition test showed
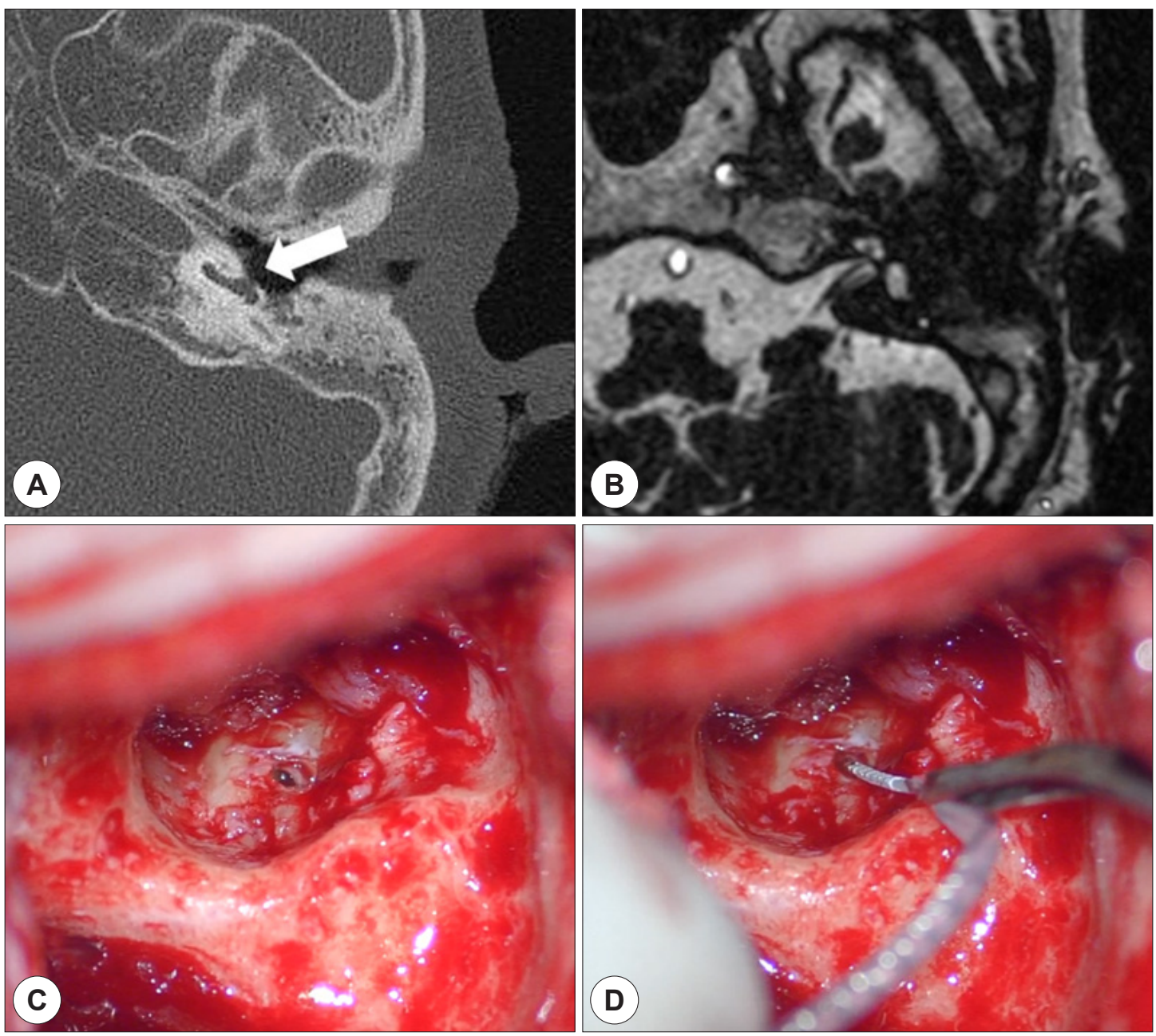

Fig. 2. Preoperative imaging and Intraoperative finding in case 2. A : Axial CT scan showing cochlear fistula. White arrow indicates the cochlear fistula. B : Magnetic resonance imaging (MRI) showed no abnormalities in the left inner ear or cochlear nerve. C : Cochlear fistula after removal of mucosa of middle ear. D : Electrode insertion via cochlear fistula. 
90\% discrimination No signs of meningitis or recurrent COM was observed at postoperative 12 months.

\section{Discussion}

Cochlear fistula caused by COM is rare; to date, there has been one report of CI that performed sequentially after cochlear fistula repair with or without subtotal petrosectomy. ${ }^{5)}$ In the present report, both the patients had inactive COM, and the inflammation could be removed completely with subtotal petrosectomy. Thereafter, we performed simultaneous inflammation removal, fistula repair, and $\mathrm{CI}$ in a single step, with good hearing outcomes. And also there was no evidence of Cholesteatoma on the CT scan and operative finding. The most main concern in single stage surgery is that there would be a risk that inflammation could spread along the electrode or through the cochlear fistula, causing complications such as meningitis or recurrent COM. However, single stage would be preferred when subtotal petrosectomy was conducted during $\mathrm{CI}$ in $\mathrm{COM}$, considering the fact that the incidence of residual and/or recurrent cholesteatoma in the obstructed cavity was considerably low and the middle ear cavity was completely isolated from the external environment and the nasal cavity. Meanwhile, depending on the location and size of the cochlear fistula, the fistula may be repaired or directly inserted with the electrode. In the first patient, it was repaired with cartilage, and the electrode was inserted to the round window. However, in the second patient, the cochlea fistula was relatively small in size compared to the diameter of the electrode and located anterior to the round window, which could be inserted in the scala tympani. Therefore, the electrode was inserted to the cochlear fistula so as to not exert additional injuries on the cochlea.

In conclusion, simultaneous subtotal petrosectomy and $\mathrm{CI}$ in patients with a cochlear fistula could be an efficient and effective treatment method. However, the clinical approach should be customized for each patient based on the clinical findings and level of middle ear inflammation.

This work was supported by clinical research grant from Pusan National University Hospital in 2020.

\section{REFERENCES}

1) Jung HW, Rhee CS, Lee JH, Kwon TY. Cochlear fistula: report of 2 cases. Korean J Otorhinolaryngol-Head Neck Surg 1997;40(9):1344-7.

2) Postelmans JT, Stokroos RJ, Linmans JJ, Kremer B. Cochlear implantation in patients with chronic otitis media: 7 years' experience in Maastricht. Eur Arch Otorhinolaryngol 2009;266(8):1159-65.

3) Issing PR, Schonermark MP, Winkelmann S, Kempf HG, Ernst A. Cochlear implantation in patients with chronic otitis: indications for subtotal petrosectomy and obliteration of the middle ear. Skull Base Surg 2006;8(3):127-31.

4) Hellingman CA, Dunnebier EA. Cochlear implantation in patients with acute or chronic middle ear infectious disease: a review of the literature. Eur Arch Otorhinolaryngol 2009;266(2):171-6.

5) Kang WS, Song CI, Yoon TH. Cochlear implantations in patients with Cochlear fistulae. Otolaryngol Head Neck Surg 2010;143(3):463-4.

6) Yoon YH, Lee JB, Chung JH, Park KW, Kim BJ, Choi JW. Cochlear implantation in patients with chronic suppurative otitis media: surgical outcomes and a management algorithm. Audiol Neurootol 2020;25:151-7. 